\title{
A DICHOTOMY THEOREM FOR THE ADJOINT OF A SEMIGROUP OF OPERATORS
}

\author{
J. M. A. M. VAN NEERVEN
}

(Communicated by Palle E. T. Jorgensen)

\begin{abstract}
Let $T(t)$ be a $C_{0}$-semigroup of linear operators on a Banach space $X$, and let $X^{\otimes}$, resp. $X^{\odot}$, denote the closed subspaces of $X^{*}$ consisting of all functionals $x^{*}$ such that the map $t \mapsto T^{*}(t) x^{*}$ is strongly continuous for $t>0$, resp. $t \geq 0$.

Theorem. Every nonzero orbit of the quotient semigroup on $X^{*} / X^{\otimes}$ is nonseparably valued. In particular, orbits in $X^{*} / X^{\odot}$ are either zero for $t>0$ or nonseparable. It also follows that the quotient space $X^{*} / X^{\otimes}$ is either zero or nonseparable. If $T(t)$ extends to a $C_{0}$-group, then $X^{*} / X^{\odot}$ is either zero or nonseparable.
\end{abstract}

For the proofs we make a detailed study of the second adjoint of a $C_{0^{-}}$semigroup.

\section{INTRODUCTION}

The basic difficulty in adjoint semigroup theory is that the adjoint $T^{*}(t)$ of a $C_{0}$-semigroup $\{T(t)\}_{t \geq 0}$ (briefly, $\left.T(t)\right)$ of linear operators on a Banach space $X$ need not be strongly continuous on $X^{*}$ again. Therefore, we define

$$
X^{\otimes}:=\left\{x^{*} \in X^{*}: \text { the map } t \mapsto T^{*}(t) x^{*} \text { is strongly continuous for } t>0\right\} .
$$

Similarly we define $X^{\odot}$ by replacing ' $t>0$ ' by ' $t \geq 0$ '. Both are closed $T^{*}(t)$ invariant subspaces of $X^{*}$. Clearly $X^{\odot} \subset X^{\otimes}$, and if $T(t)$ is a $C_{0}$-group, then $X^{\odot}=X^{\otimes}$, but in general the inclusion may be proper. The main result of this paper is the following theorem, which describes the orbits of the quotient semigroup on $X^{*} / X^{\otimes}$.

Theorem 0.1. Let $T(t)$ be a $C_{0}$-semigroup on a Banach space $X$. The orbit under the quotient semigroup of every nonzero element of $X^{*} / X^{\otimes}$ is nonseparably valued.

By Pettis's measurability theorem and the Measurable Semigroup Theorem (see $\S 1$ ) it is trivial that if the $T^{*}(t)$-orbit of an $x^{*} \in X^{*}$ is not strongly continuous for $t>0$ (i.e., the class of $x^{*}$ in $X^{*} / X^{\otimes}$ is nonzero), then the orbit

Received by the editors November 24, 1991.

1991 Mathematics Subject Classification. Primary 47D03.

Key words and phrases. Adjoint semigroup, $C_{0}$-semigroup, Baire-1 functional, Pettis integral, weakly Borel measurable. 
of $x^{*}$ under $T^{*}(t)$ is nonseparably valued. The content of the main theorem is that this nonseparability is caused by the bad 'part' of $X^{*}$, viz. $X^{*} / X^{\otimes}$, and not by the good part $X^{\otimes}$. This is not at all evident, since one must rule out the possibility that a nonseparable orbit is so 'parallel' to a nonseparable $X^{\otimes}$ that on the one hand its nonseparability is due to nonseparability of $X^{\otimes}$, whereas on the other hand, upon dividing out $X^{\otimes}$, the quotient orbit becomes separable.

We note some immediate corollaries of the main theorem.

Corollary 0.2. The quotient space $X^{*} / X^{\otimes}$ is either zero or nonseparable.

That some result in this direction should be true was conjectured by Ben de Pagter (personal communication). We remark that it is not true that $X^{*} / X^{\odot}$ is always zero or nonseparable (see [Ne2] for an example of a $C_{0}$-semigroup on $c_{0}$ with $\left.\operatorname{dim} c_{0}^{*} / c_{0}^{\odot}=1\right)$. However, if $X^{*} / X^{\odot}$ is separable, then one sees from Corollary 0.2 that $X^{*}=X^{\otimes}$. This solves affirmatively a question raised in [Ne1]. Moreover, if $T(t)$ extends to a $C_{0}$-group, then $X^{\otimes}=X^{\odot}$, and it follows that $X^{*} / X^{\odot}$ is either zero or nonseparable.

By applying the natural map $X^{*} / X^{\odot} \rightarrow X^{*} / X^{\otimes}$ to a separably valued orbit in $X^{*} / X^{\odot}$, Theorem 0.1 implies the following dichotomy result.

Corollary 0.3. Every quotient orbit in $X^{*} / X^{\odot}$ is either zero for $t>0$ or nonseparably valued.

We will give a detailed proof of Corollary 0.2 rather than Theorem 0.1 . After that we show how the argument has to be modified in order to deal with the general case. The reason for proceeding in this way is to make the main ideas in the proof more transparent. Let us briefly sketch them. We assume that $X^{*} / X^{\otimes}$ is separable and want to prove that it is zero. Identifying the dual of $X^{*} / X^{\otimes}$ with the annihilator $X^{\otimes \perp}$ of $X^{\otimes}$ in $X^{* *}$, we show that $X^{\otimes \perp} \cap \mathscr{B}_{1}(X)$ separates the points of the quotient space. Here $\mathscr{B}_{1}(X)$ is the subspace of $X^{* *}$ of all so-called Baire- 1 functionals. The functionals in this intersection give rise to functionals on the quotient space which have the additional property that the quotient semigroup is measurable with respect to them. Using this, it follows that the quotient semigroup is strongly continuous for $t>0$ with respect to some weaker norm. This is shown to imply that there is a nonzero Baire-1 functional in $\left(X^{*} / X^{\otimes}\right)^{\odot}$ if $X^{*} / X^{\otimes}$ is nonzero. The proof will then be complete if we can show that such functionals do not exist. This is essentially proved in $\S 2$ and requires a detailed study of the strong continuity of the second adjoint semigroup $T^{* *}(t)$ on $X^{* *}$.

\section{Preliminaries}

We assume the reader to be familiar with the basics of the theory of semigroups of linear operators. We will use the following Measurable Semigroup Theorem [HPh, Theorem 10.2.3]: If $T(t)$ is a semigroup such that $t \mapsto T(t) x$ is strongly measurable for each $x \in X$, then $T(t)$ is actually strongly continuous for $t>0$ (briefly, $T(t)$ is $C_{>0}$ ). We also assume familiarity with the elementary theory of adjoint semigroups. A nice introduction with detailed proofs is given in [BB]. To fix the notation we repeat some definitions now.

The adjoint of a (not necessarily $C_{0}$ ) semigroup $T(t)$ on a Banach space $X$ is the semigroup $T^{*}(t)$ on the dual space $X^{*}$ obtained by taking pointwise in $t$ 
the adjoint operators $T^{*}(t):=(T(t))^{*}$. The semigroup dual of $X$ with respect to $T(t)$ is the linear subspace

$$
X^{\odot}:=\left\{x^{*} \in X^{*}: \lim _{t \downarrow 0}\left\|T^{*}(t) x^{*}-x^{*}\right\|=0\right\} .
$$

In other words, $X^{\odot}$ is the largest subspace of $X^{*}$ on which $T^{*}(t)$ acts in a strongly continuous way, and clearly $X^{\odot}$ is $T(t)$-invariant. If $T(t)$ is locally bounded at $t=0$, e.g., if $T(t)$ is of class $C_{0}$, then $X^{\odot}$ is closed.

For the rest of this paper we assume that $T(t)$ is a $C_{0}$-semigroup on $X$. For such semigroups we have $X^{\odot}=\overline{D\left(A^{*}\right)}$ where $A^{*}$ is the adjoint of the infinitesimal generator $A$ of $T(t)$. Thus $X^{\odot}$ is precisely the norm closure of the range of the adjoint $R(\lambda, A)^{*}=R\left(\lambda, A^{*}\right)$ of the resolvent of $A$. An $x^{*} \in X^{*}$ belongs to $X^{\odot}$ if and only if $\lim _{\lambda \rightarrow \infty} \lambda R\left(\lambda, A^{*}\right) x^{*}=x^{*}$ strongly. $X^{\odot}$ induces an equivalent norm in $X$, so in particular $X^{\odot}$ is weak *-dense in $X^{*}$, and hence if $X$ is reflexive we have $X^{\odot}=X^{*}$. From elementary examples one sees that in general, however, $X^{\odot}$ is a proper subspace of $X^{*}$.

Next we will define the second adjoint of a $C_{0}$-semigroup. Since $X^{\odot}$ is $T^{*}(t)$-invariant, the restrictions $T^{\odot}(t)$ of $T^{*}(t)$ to $X^{\odot}$ define a $C_{0}$-semigroup on $X^{\odot}$. Starting from the semigroup $T^{\odot}(t)$ we can define $X^{\odot \odot}:=\left(X^{\odot}\right)^{\odot}$, the subspace of strong continuity of $T^{\odot *}(t)=\left(T^{\odot}(t)\right)^{*}$. Define a map $j: X \rightarrow$ $X^{\odot *}$,

$$
\left\langle j x, x^{\odot}\right\rangle:=\left\langle x^{\odot}, x\right\rangle .
$$

Since $X^{\odot}$ induces an equivalent norm in $X$, the map $j$ is an embedding into, and in fact maps $X$ into $X \odot \odot$. In this way we can identifiy $X$ with a closed subspace of $X^{\odot \odot}$. One has to be a little bit careful here, since $j$ is in general not isometric. If $j X=X \odot \odot$, then $X$ is said to be $\odot$-reflexive with respect to $T(t)$. This is the case if and only if the resolvent $R(\lambda, A)$ is weakly compact [Pa].

In turn the space $X^{\odot \odot}$ can be identified in a natural way with a closed subspace of $X^{* *}$ in the following way [Cl1, Part IV].

Lemma 1.1. The limit

$$
\left\langle k x^{\odot \odot}, x^{*}\right\rangle=\lim _{\lambda \rightarrow \infty}\left\langle x^{\odot \odot}, \lambda R\left(\lambda, A^{*}\right) x^{*}\right\rangle
$$

exists for all $x^{\odot \odot} \in X^{\odot \odot}$ and defines an isomorphical embedding $k: X \odot \odot \rightarrow$ $X^{* *}$. Moreover, for $x^{\odot \odot} \in X^{\odot \odot}$ and $x^{\odot} \in X^{\odot}$ we have $\left\langle k x^{\odot \odot}, x^{\odot}\right\rangle=$ $\left\langle x^{\odot \odot}, x^{\odot}\right\rangle$, and if $i: X \rightarrow X^{* *}$ is the natural map, then we have $i=k \circ j$.

There is a second natural way to define a second semigroup dual space, viz. by starting from $T^{*}(t)$ rather than from $T^{\odot}(t)$ :

$$
X_{\odot \odot}:=\left\{x^{* *} \in X^{* *}: \lim _{t \downarrow 0}\left\|T^{* *}(t) x^{* *}-x^{* *}\right\|=0\right\} .
$$

Thus, if we understand $X^{*}$ to be equipped with the semigroup $T^{*}(t)$, then $X_{\odot \odot}$ is precisely $\left(X^{*}\right)^{\odot}$.

It is easy to see that $k X^{\odot \odot} \subset X_{\odot \odot}$, and it is natural to ask whether $k X^{\odot} \odot$ actually equals $X_{\odot \odot}$. That, in general, this is not the case is shown by the following example. Another example is given in [C12]. We need some terminology and elementary facts about Banach lattices which can be found in [S]. 
Example 1.2. Let $T(t)$ be the rotation group on $X=C(T)$ defined by

$$
T(t) f\left(e^{i \theta}\right)=f\left(e^{i(t+\theta)}\right) .
$$

Then $X^{\odot \odot}=X=C(T)$. We will construct a nonzero $x_{\odot \odot} \in X_{\odot \odot}$ which annihilates $X^{\odot}$, so $x_{\odot \odot} \notin k X^{\odot \odot}$. Let $x^{* *} \in X^{* *}$ be any nonzero vector annihilating the subspace $L^{1}(T)=X^{\odot}$ of $X^{*}$. Regarding $X^{* *}$ as a Banach lattice by replacing $x^{* *}$ by its modulus $\left|x^{* *}\right|$, which also annihilates $X^{\odot}$, we may assume without loss of generality that $x^{* *} \geq 0$. Put

$$
x_{\odot \odot}:=\sup _{s \in \mathbb{R}} T^{* *}(s) x^{* *}
$$

Since $X^{* *}$ is a $C(K)$-space by the Kakutani-Krein representation theorem and since the orbit of $x^{* *}$ is norm bounded, this orbit is order bounded. Since $X^{* *}$, being a dual space, is Dedekind complete, it follows that the supremum indeed exists. Trivially $x_{\odot \odot} \geq x^{* *}$, so $x_{\odot \odot} \neq 0$. Since for each $t \geq 0$ the operator $T^{* *}(t)$ is an order-continuous lattice homomorphism, we have

$$
T^{* *}(t) x_{\odot \odot}=T^{* *}(t)\left(\sup _{s \in \mathbb{R}} T^{* *}(s) x^{* *}\right)=\sup _{s \in \mathbb{R}} T^{* *}(t+s) x^{* *}=x_{\odot \odot} .
$$

Hence $x_{\odot \odot} \in X_{\odot \odot}$. On the other hand, since $x^{* *}$ annihilates $X^{\odot}$, so does each $T^{* *}(t) x^{* *}$. Since the annihilator of the projection band $X^{\odot}$ is a (projection) band in $X^{* *}$, it follows that $x_{\odot \odot}$ annihilates $X^{\odot}$ as well.

The construction in this example can be carried out in much more generality. In fact, for a positive $C_{0}$-semigroup on a space $X=C(K), K$ compact Hausdorff, one can prove that the following assertions are equivalent:

(1) $X_{\odot \odot}=k X^{\odot \odot}$.

(2) $T^{*}(t)$ is $C_{>0}$.

(3) $T^{*}(t)$ is weakly Borel measurable.

The proof takes us too far afield and will be given elsewhere.

We assume the reader to be familiar with the notions of strong measurability and the Bochner-, weak*-, and Pettis integral. For the definitions and basic properties we refer to [DU]. The following lemma is easily checked by direct computation.

Lemma 1.3. Let $S(t)$ be a semigroup (not necessarily $C_{0}$ ) on $X$. Suppose the map $t \mapsto S^{*}(t) x^{*}$ is locally bounded at $t=0$ and weak*-measurable $(e . g ., S(t)$ is $\left.C_{0}\right)$. Then $w^{*} \int_{0}^{t} S^{*}(\sigma) x^{*} d \sigma \in X^{\odot}$ for all $t>0$.

\section{THE SPACES $X \odot \odot$ AND $X_{\odot} \odot$}

Throughout this and the following section $T(t)$ is a $C_{0}$-semigroup on a Banach space $X$. We will be concerned with the spaces $X \odot \odot$ and $X_{\odot \odot}$, whose properties play a key role in the proof of Theorem 0.1 . The guiding line in this section will be to try to understand why $k X^{\odot \odot}$ can be a proper subspace of $X_{\odot \odot}$. The first main result in this direction will be Theorem 2.4, which shows that $k X^{\odot \odot}$ is complemented in $X_{\odot \odot}$ and that its complement is precisely $\left(X^{*} / X^{\odot}\right)^{\odot}$. This establishes a connection between the theories of $X_{\odot \odot}$ and that of $X^{*} / X^{\odot}$.

Let $r: X^{* *} \rightarrow X^{\odot *}$ denote the restriction map, i.e., $\left\langle r x^{* *}, x^{\odot}\right\rangle:=\left\langle x^{* *}, x^{\odot}\right\rangle$ $\forall X^{\odot} \in X^{\odot}$. It is easily checked that $r$ maps $X_{\odot \odot}$ into $X^{\odot \odot}$. 
Lemma 2.1. $k \circ r: X_{\odot \odot} \rightarrow k X^{\odot \odot}$ is a projection of $X_{\odot \odot}$ onto $k X^{\odot \odot}$.

Proof. Since $k X^{\odot \odot} \subset X_{\odot \odot}$, it suffices to prove that $k r(k x \odot \odot)=k x \odot \odot$ holds for all $x^{\odot \odot} \in X^{\odot \odot}$. But this follows from the obvious fact that $r k x^{\odot \odot}=x^{\odot \odot}$ for all $x^{\odot \odot} \in X^{\odot \odot}$.

The projection on $X_{\odot \odot}$ described above will be denoted by $\pi$.

In order to identify the complement of $k X^{\odot \odot}$ in $X_{\odot \odot}$ we need some results on quotient semigroups. Let $S(t)$ be a semigroup on $X$, not necessarily $C_{0}$. Let $Y$ be a closed $S(t)$-invariant subspace, and let $q: X \rightarrow X / Y$ be the quotient map. The quotient semigroup on $X / Y$ is defined by

$$
S_{q}(t) q x=q(S(t) x) .
$$

The dual space $(X / Y)^{*}$ is naturally isometrically isomorphic to the annihilator $Y^{\perp}:=\left\{x^{*} \in X^{*}:\left\langle x^{*}, y\right\rangle=0, \forall y \in Y\right\}$ of $Y$; the isomorphism $m:(X / Y)^{*} \rightarrow$ $Y^{\perp}$ is given by $\left\langle m z^{*}, x\right\rangle:=\left\langle z^{*}, q x\right\rangle, z^{*} \in(X / Y)^{*}$.

Lemma 2.2. For any $z^{*} \in(X / Y)^{*}$ we have $\left\langle m\left(S_{q}^{*}(t) z^{*}\right)\right\rangle=S^{*}(t)\left(m z^{*}\right)$.

Proof. Let $x \in X$ be arbitrary. Then

$$
\begin{aligned}
\left\langle m\left(S_{q}^{*}(t) z^{*}\right), x\right\rangle & =\left\langle S_{q}^{*}(t) z^{*}, q x\right\rangle=\left\langle z^{*}, S_{q}(t) q x\right\rangle \\
& =\left\langle z^{*}, q(S(t) x)\right\rangle=\left\langle m z^{*}, S(t) x\right\rangle=\left\langle S^{*}(t)\left(m z^{*}\right), x\right\rangle .
\end{aligned}
$$

Thus we may identify $S_{q}^{*}(t)$ with the restriction of $S^{*}(t)$ to $Y^{\perp}$. This proves

Lemma 2.3. The map $m$ induces an isometrical isomorphism $(X / Y)^{\odot} \simeq X^{\odot} \cap$ $Y^{\perp}$.

From now on, let $T(t)$ again be a $C_{0}$-semigroup. Let $q: X^{*} \rightarrow X^{*} / X^{\odot}$ be the quotient map, and let $T_{q}(t)$ be the quotient semigroup on $X^{*} / X^{\odot}$. From $\left\|T_{q}(t)\right\| \leq\left\|T^{*}(t)\right\|$ it follows that $T_{q}(t)$ is a locally bounded semigroup again. Let $T_{q}^{*}(t)$ denote the adjoint semigroup $\left(T_{q}(t)\right)^{*}$. Let $m:\left(X^{*} / X^{\odot}\right)^{*} \rightarrow X^{\odot \perp}$ be the natural isomorphism. In this way $\left(X^{*} / X^{\odot}\right)^{\odot}$ is identified with a closed subspace of $X^{* *}$.

Theorem 2.4. $X_{\odot \odot}=k X^{\odot \odot} \oplus m\left(X^{*} / X^{\odot}\right) \odot$.

Proof. By Lemma 2.1 we have $X_{\odot \odot}=k X^{\odot \odot} \oplus Y$, where $Y=\operatorname{ker} \pi$. We claim that $\operatorname{ker} \pi=X_{\odot \odot} \cap X^{\odot \perp}$. Indeed, it is clear from $X^{\odot \perp} \subset \operatorname{ker} r$, and the definition of $\pi$ shows that $X_{\odot \odot} \cap X^{\odot \perp} \subset \operatorname{ker} \pi$. Conversely, if $x_{\odot \odot} \in \operatorname{ker} \pi$, then since $k$ is an isomorphism into we must have $r x_{\odot \odot}=0$, which means that $x_{\odot \odot} \in X^{\odot \perp}$. This proves the claim. From Lemma 2.3 we know that $m\left(X^{*} / X^{\odot}\right)^{\odot}=\left(X^{*}\right)^{\odot} \cap X^{\odot \perp}=X_{\odot \odot} \cap X^{\odot \perp}$. Therefore ker $\pi=m\left(X^{*} / X^{\odot}\right)^{\odot}$.

In the next section we will be concerned with the space $X^{\otimes}$. At this point it is interesting to note that one can prove that there are natural isomorphisms $X^{\odot \odot} \simeq X^{\otimes \odot}$ and $\left(X^{*} / X^{\odot}\right)^{\odot} \simeq\left(X^{*} / X^{\otimes}\right)^{\odot}$.

With the information of Theorem 2.4 we can characterise $k X^{\odot \odot}$ as those elements of $X_{\odot \odot}$ that commute with the weak *-integral of the adjoint semigroup.

Corollary 2.5. An element $x_{\odot \odot} \in X_{\odot \odot}$ belongs to $k X^{\odot} \odot$ if and only if for all $t>0$ and $x^{*} \in X^{*}$ we have

$$
\left\langle x_{\odot \odot}, w^{*} \int_{0}^{t} T^{*}(\sigma) x^{*} d \sigma\right\rangle=\int_{0}^{t}\left\langle x_{\odot \odot}, T^{*}(\sigma) x^{*}\right\rangle d \sigma .
$$


Proof. The 'only if' part is proved in [Cl1]. For the 'if' part, let $x_{\odot \odot} \in X_{\odot \odot}$ such that $(2.1)$ holds for all $t$ and $x^{*}$. Write $x_{\odot \odot}=k x^{\odot \odot}+y$ with $y \in \operatorname{ker} \pi$. Since (2.1) holds for $k x \odot \odot$ by the above discussion, it follows that (2.1) also holds for $y$. Let $x^{*} \in X^{*}$ be fixed. By Lemma 1.3, for all $t>0$ we have

$$
\left\langle y, w^{*} \int_{0}^{t} T^{*}(\sigma) x^{*} d \sigma\right\rangle=0 .
$$

Thus, for all $t>0$,

$$
\int_{0}^{t}\left\langle T^{* *}(\sigma) y, x^{*}\right\rangle d \sigma=\int_{0}^{t}\left\langle y, T^{*}(\sigma) x^{*}\right\rangle d \sigma=0 .
$$

But $t \mapsto\left\langle T^{* *}(t) y, x^{*}\right\rangle$ is a continuous function for $t \geq 0$ since $y \in X_{\odot \odot}$, so it must be identically zero. In particular, $\left\langle y, x^{*}\right\rangle=0$. Since $x^{*}$ was arbitrary, it follows that $y=0$, as was to be shown.

Corollary 2.6. $X_{\odot \odot}=k X^{\odot \odot}$ if and only if for all $t>0, x^{*} \in X^{*}$, and $x_{\odot \odot} \in X_{\odot \odot}$ we have

$$
\left\langle x_{\odot \odot}, w^{*} \int_{0}^{t} T^{*}(\sigma) x^{*} d \sigma\right\rangle=\int_{0}^{t}\left\langle x_{\odot \odot}, T^{*}(\sigma) x^{*}\right\rangle d \sigma .
$$

In particular, $X_{\odot \odot}=k X^{\odot \odot}$ if $T^{*}(t)$ is $C_{>0}$ or, more generally, if $T^{*}(t)$ is Pettis integrable. Thus the discrepancy of $X^{\odot \odot}$ and $X_{\odot \odot}$ is due to the fact that $T^{*}(t)$, being weak*-integrable, may fail to be Pettis integrable.

It is not hard to show that a semigroup which is uniformly continuous for $t>0$ is $\odot$-reflexive if and only if it is weakly compact for $t>0$. This generalises as follows:

Theorem 2.7. For a $C_{0}$-semigroup $T(t)$ the following assertions are equivalent:

(1) $T(t)$ is weakly compact.

(2) $X$ is $\odot$-reflexive with respect to $T(t)$, and $T^{* *}(t)$ is $C_{>0}$.

Proof. Suppose first that $T(t)$ is weakly compact. Then $T^{* *}(t) X^{* *} \subset X$ for each $t>0$ by Gantmacher's theorem. Trivially this implies that $T^{* *}(t)$ is $C_{>0}$. Fix $x^{\odot \odot} \in X^{\odot \odot}$. Then $T^{* *}(t) k x \odot \odot \subset X$ for all $t>0$, and letting $t \downarrow 0$ it follows from the inclusion $k X^{\odot \odot} \subset X_{\odot \odot}$ that $k x^{\odot \odot} \in X$, so $X$ is $\odot$-reflexive.

Conversely, suppose that (2) holds. By assumption on $T^{* *}(t), T^{*}(t)$ is weakly continuous for $t>0$. Fix $t_{0}>0$ and $x^{*} \in X^{*}$. Then $T^{*}(t)$ is weakly continuous, hence strongly continuous, on the closed linear span of $\left\{T^{*}(t) x^{*}\right.$ : $\left.t \geq t_{0}\right\}$. It follows that $T^{*}(t)$ is $C_{>0}$, so by Corollary 2.6 and $\odot$-reflexivity we have $X_{\odot \odot}=k X^{\odot \odot}=X$. Since $T^{* *}(t)$ is $C_{>0}$, we have $T^{* *}(t) X^{* *} \subset X_{\odot \odot}$ for all $t>0$. Hence $T^{* *}(t) X^{* *} \subset X$ for all $t>0$, and the weak compactness of $T(t)$ follows from Gantmacher's theorem.

Recall that an element $x^{* *} \in X^{* *}$ is called a Baire-1 functional if it is the weak *-limit in $X^{* *}$ of a sequence $\left(x_{n}\right) \subset X$. The set of all Baire-1 functionals is a linear subspace of $X^{* *}$ and is denoted by $\mathscr{B}_{1}(X)$. A trivial but useful consequence of Corollary 2.6 and the dominated convergence theorem is the following corollary, which will be used in Lemma 3.6. 
Corollary 2.8. $X_{\odot \odot} \cap \mathscr{B}_{1}(X) \subset k X^{\odot \odot ~ . ~}$

From Corollary 2.6 we know that $X_{\odot \odot}=k X^{\odot \odot}$ if $T^{*}(t)$ is $C_{>0}$. The next result shows that in fact a much weaker condition will do if $X$ is separable. For this we use the following deep result of Riddle, Saab, and Uhl [RSU]: If $K$ is compact Hausdorff and $X$ is a separable Banach space and $\psi: K \rightarrow X^{*}$ is a bounded, universally weakly measurable function, then $\psi$ is universally Pettis integrable. In particular, if $T^{*}(t)$ is weakly Borel measurable, it follows from this theorem that $T^{*}(t)$ is actually Pettis integrable. Combining this with Corollary 2.6 we obtain

Corollary 2.9. Suppose $X$ is separable. If $T^{*}(t)$ is weakly Borel measurable, then $X_{\odot \odot}=k X^{\odot \odot}$.

\section{Proof of Theorem 0.1}

We will write $X_{\otimes}$ for $X^{*} / X^{\otimes}$. The quotient semigroup of $T^{*}(t)$ on $X_{\otimes}$ will be denoted by $T_{\otimes}(t)$. Often we will identify $\left(X_{\otimes}\right)^{*}$ with $X^{\otimes \perp}$.

Lemma 3.1. If $X_{\otimes}$ is separable, then for every nonzero $x_{\otimes} \in X_{\otimes}$ there is a Baire-1 functional $x^{\otimes \perp} \in X^{\otimes \perp}$ such that $\left\langle x^{\otimes \perp}, x_{\otimes}\right\rangle \neq 0$.

Proof. Fix a nonzero $x_{\otimes} \in X_{\otimes}$, and let $x^{*} \in X^{*}$ be a representant of $x_{\otimes}$. Since $x^{*} \notin X^{\otimes}$, there is an $\epsilon>0, t>0$, and a sequence $t_{n} \downarrow 0$ such that, for all $n$,

$$
\left\|T^{*}\left(t+t_{n}\right) x^{*}-T^{*}(t) x^{*}\right\|>\epsilon .
$$

Choose norm one elements $x_{n} \in X$ such that

$$
\left|\left\langle T^{*}\left(t+t_{n}\right) x^{*}-T^{*}(t) x^{*}, x_{n}\right\rangle\right|>\epsilon,
$$

and put $z_{n}:=T\left(t+t_{n}\right) x_{n}-T(t) x_{n}$. The sequence $\left(z_{n}\right)$ is bounded, and for all $x^{\otimes} \in X^{\otimes}$ we have

$$
\begin{aligned}
\lim _{n \rightarrow \infty}\left|\left\langle x^{\otimes}, z_{n}\right\rangle\right| & =\lim _{n \rightarrow \infty}\left|\left\langle T^{*}\left(t+t_{n}\right) x^{\otimes}-T^{*}(t) x^{\otimes}, x_{n}\right\rangle\right| \\
& \leq \limsup _{n \rightarrow \infty}\left\|T^{*}\left(t+t_{n}\right) x^{\otimes}-T^{*}(t) x^{\otimes}\right\|=0 .
\end{aligned}
$$

Furthermore, since $X_{\otimes}$ is separable, there is a countable set $F \subset X^{*}$ such that the linear span of $X^{\otimes} \cup F$ is norm dense in $X^{*}$. By a diagonal argument we find a subsequence of $\left(z_{n}\right)$, relabelled $\left(z_{n}\right)$ again, such that $\lim _{n \rightarrow \infty}\left\langle f^{*}, z_{n}\right\rangle$ exists for all $f^{*} \in F$. Passing once more to a subsequence if necessary we also may assume that

$$
\lim _{n \rightarrow \infty}\left|\left\langle x^{*}, z_{n}\right\rangle\right|=\lim _{n \rightarrow \infty}\left|\left\langle T^{*}\left(t+t_{n}\right) x^{*}-T^{*}(t) x^{*}, x_{n}\right\rangle\right|=: \epsilon_{0} \geq \epsilon .
$$

Regarding $\left(z_{n}\right)$ as a (bounded) sequence in $X^{* *}$, it follows that $\left(z_{n}\right)$ is weak * convergent to some $x^{\otimes \perp} \in X^{* *}$. By (3.1) we have $x^{\otimes \perp} \in X^{\otimes \perp}$, and this together with (3.2) implies that

$$
\left|\left\langle x^{\otimes \perp}, x_{\otimes}\right\rangle\right|=\left|\left\langle x^{\otimes \perp}, x^{*}\right\rangle\right|=\epsilon_{0}>0 .
$$

Let $\Gamma \subset X^{* *}$ be the linear subspace $X^{\otimes \perp} \cap \mathscr{B}_{1}(X)$. By Lemma 3.1, $\Gamma$ separates the points of $X_{\otimes}$, but the problem is that we do not know whether $\Gamma$ induces an equivalent norm on $X_{\otimes}$. However, this causes only small technical 
complications with which we shall deal next. Define a norm $|\cdot|$ on $X_{\otimes}$ by putting

$$
\left|x_{\otimes}\right|:=\sup _{x^{\otimes \perp} \in \Gamma,\left\|x^{\otimes \perp}\right\| \leq 1}\left|\left\langle x^{\otimes \perp}, x_{\otimes}\right\rangle\right| .
$$

This is indeed a norm, since, by Lemma 3.1, $\left|x_{\otimes}\right|=0$ implies $x_{\otimes}=0$. Note that $\left|x_{\otimes}\right| \leq\left\|x_{\otimes}\right\|$ for all $x_{\otimes} \in X_{\otimes}$. In this way $\left(X_{\otimes},|\cdot|\right)$ becomes a normed linear space; denote its completion by $\overline{X_{\otimes}}$. Then $\overline{X_{\otimes}}$ is a Banach space, and trivially each $x^{\otimes \perp} \in \Gamma$ of $\|\cdot\|$-norm $\leq 1$ extends to a bounded linear functional on $\overline{X_{\otimes}}$ of $|\cdot|$-norm $\leq 1$. Our next aim is to show that $T_{\otimes}(t)$ extends to a semigroup on $\overline{X_{\otimes}}$. This follows from a density argument and the following lemma, which uses the obvious fact that $\Gamma$ is $T^{* *}(t)$-invariant.

Lemma 3.2. $\left|T_{\otimes}(t)\right| \leq\|T(t)\|$.

Proof. Let $\left|x_{\otimes}\right|=1$. Then

$$
\begin{aligned}
\left|T_{\otimes}(t) x_{\otimes}\right| & =\sup _{x^{\otimes \perp} \in B_{\Gamma}}\left|\left\langle x^{\otimes \perp}, T_{\otimes}(t) x_{\otimes}\right\rangle\right|=\sup _{x_{\otimes} \in B_{\Gamma}}\left|\left\langle T^{* *}(t) x^{\otimes \perp}, x_{\otimes}\right\rangle\right| \\
& \leq \sup _{z^{\otimes \perp} \in\left\|T^{* * *}(t)\right\| \cdot B_{\Gamma}}\left|\left\langle z^{\otimes \perp}, x_{\otimes}\right\rangle\right|=\|T(t)\| \cdot\left|x_{\otimes}\right| .
\end{aligned}
$$

Lemma 3.3. $T_{\otimes}(t)$ is $C_{>0}$ with respect to $|\cdot|$.

Proof. Denote the extension of $T_{\otimes}(t)$ to $\overline{X_{\otimes}}$ by $\overline{T_{\otimes}(t)}$. First, $\Gamma \subset\left(\overline{X_{\otimes}}\right)^{*}$ is norming for $\overline{X_{\otimes}}$. Second, since $\Gamma \subset \mathscr{B}_{1}(X)$, for each $x^{\otimes \perp} \in X^{\otimes \perp}$ and $x_{\otimes} \in X_{\otimes}$, the function

$$
t \mapsto\left\langle x^{\otimes \perp}, \overline{T_{\otimes}(t)} x_{\otimes}\right\rangle=\left\langle x^{\otimes \perp}, T_{\otimes}(t) x_{\otimes}\right\rangle
$$

is the pointwise limit of a sequence of continuous functions

$$
t \mapsto\left\langle T^{*}(t) x^{*}, z_{n}\right\rangle,
$$

where $q x^{*}=x_{\otimes}$ and $z_{n} \rightarrow x^{\otimes \perp}$ weak $^{*}$; hence, in particular, this function is measurable. Third, since the topology in $X_{\otimes}$ induced by $|\cdot|$ is coarser than the one induced by $\|\cdot\|$, and since $X_{\otimes}$ is $\|\cdot\|$-separable, for each $x_{\otimes} \in X_{\otimes}$ the function $t \rightarrow T_{\otimes}(t) x_{\otimes}$ is $|\cdot|$-separably valued. Combining these three observations with Pettis's Measurability Theorem shows that $t \rightarrow T_{\otimes}(t) x_{\otimes}$ is $|\cdot|$-strongly measurable for each $x_{\otimes} \in X_{\otimes}$. By denseness the semigroup $\overline{T_{\otimes}(t)}$ is $|\cdot|$-strongly measurable for each $x_{\otimes} \in \overline{X_{\otimes}}$ and therefore $C_{>0}$ by the Measurable Semigroup Theorem.

The functionals produced in Lemma 3.1 lie in $X^{\otimes \perp}=X_{\otimes}^{*}$ but in general do not lie in $X_{\otimes}^{\odot}$. By weak *-integration we can pull them into $X_{\otimes}^{\odot}$. The next lemma describes the result.

Lemma 3.4. If $x^{\otimes \perp} \in X^{\otimes \perp} \cap \mathscr{B}_{1}(X)$, then for all $t>0$

$$
w^{*} \int_{0}^{t} T^{* *}(\sigma) x^{\otimes \perp} d \sigma=0 .
$$

Proof. First note that the weak*-integral is defined since $x^{\otimes \perp} \in \mathscr{B}_{1}(X)$. By Lemma 1.3 it is an element of $X_{\odot \odot}$. Also it belongs to $X^{\otimes \perp}$ and, hence, to $X^{\odot \perp}$. On the other hand the fact that $x^{\otimes \perp} \in \mathscr{B}_{1}(X)$, the dominated convergence theorem, and the definition of the weak*-integral imply that 
$w^{*} \int_{0}^{t} T^{* *}(\sigma) x^{\otimes \perp} d \sigma$ is in $\mathscr{B}_{1}(X)$ again. Therefore, the integral belongs to $k X^{\odot \odot}$ by Corollary 2.8. We have shown that $w^{*} \int_{0}^{t} T^{* *}(\sigma) x^{\otimes \perp} d \sigma \in k X^{\odot \odot} \cap$ $X^{\odot \perp}=\{0\}$.

Now we can prove Corollary 0.2.

Proof of Corollary 0.2. Suppose $X_{\otimes}$ is separable. If $X_{\otimes}$ were nonzero, that is, if $T^{*}(t)$ were not $C_{>0}$, then there is a $t_{0}>0$ and a $z_{\otimes} \in X_{\otimes}$ such that $x_{\otimes}:=T_{\otimes}\left(t_{0}\right) z_{\otimes} \neq 0$. Fix $x^{\otimes \perp} \in \Gamma$ such that

$$
\left\langle x^{\otimes \perp}, x_{\otimes}\right\rangle=: \epsilon>0 .
$$

By Lemma 3.3 we may choose $t>0$ so small that

$$
\left|x^{\otimes \perp}\right|\left|T_{\otimes}(\sigma) x_{\otimes}-x_{\otimes}\right|<\frac{\epsilon}{2}, \quad \forall 0 \leq \sigma \leq t .
$$

Here $\left|x^{\otimes \perp}\right|$ is the norm of $x^{\otimes \perp}$ regarded as a functional on $\overline{X_{\otimes}}$. From Lemma 3.4 we obtain

$$
\begin{aligned}
0 & =\left|\left\langle\frac{1}{t} w^{*} \int_{0}^{t} T^{* *}(\sigma) x^{\otimes \perp} d \sigma, x_{\otimes}\right\rangle\right|=\left|\frac{1}{t} \int_{0}^{t}\left\langle x^{\otimes \perp}, T_{\otimes}(t) x_{\otimes}\right\rangle d \sigma\right| \\
& \geq\left|\frac{1}{t} \int_{0}^{t}\left\langle x^{\otimes \perp}, x_{\otimes}\right\rangle d \sigma\right|-\left|\frac{1}{t} \int_{0}^{t}\left\langle x^{\otimes \perp}, T_{\otimes}(t) x_{\otimes}-x_{\otimes}\right\rangle d \sigma\right| \geq \epsilon-\frac{\epsilon}{2}=\frac{\epsilon}{2},
\end{aligned}
$$

a contradiction.

For an $x^{*} \in X^{*}$, let $Y_{\otimes}$ be the closed linear span of the orbit of $q x^{*}$ in $X_{\otimes}$, where $q: X^{*} \rightarrow X_{\otimes}=X^{*} / X^{\otimes}$ is the quotient map. It is easy to see that $Y:=q^{-1} Y_{\otimes}$ is a closed $T^{*}(t)$-invariant subspace of $X^{*}$. Now suppose that the quotient orbit of $x^{*}$ is separable in $X_{\otimes}$. Then $Y / X^{\otimes} \simeq Y_{\otimes}$ is separable. Using this observation, Theorem 0.1 can be proved by repeating word for word the proof of Corollary 0.2 , except that the roles of $X^{*}, X^{* *}, X_{\odot \odot}$, and $X_{\otimes}$ are taken over by $Y, Y^{*}, Y^{\odot}$, and $Y_{\otimes}$ respectively. To be more precise, we use the following two facts:

(i) The separability of $Y / X^{\otimes}$ allows us to prove an analogue of Lemma 3.1.

(ii) In the proof of Lemma 3.4 we did not use all the information contained in Corollary 2.8. In fact, what we used is the following: If $y \in X{ }^{\odot} \perp \cap X_{\odot \odot} \cap \mathscr{B}_{1}(X)$, then $y=0$. Letting $Y$ be as above, what we need for the proof of Theorem 0.1 is the following: If $y \in X^{\odot \perp} \cap Y^{\odot}$ is a weak*-limit (i.e., $\sigma\left(Y^{*}, Y\right)$-limit) of a sequence in $X$, then $y=0$. That this is true is proved as in Corollary 2.5 .

\section{ACKNOWLEDGMENT}

The author thanks Ben de Pagter for critically reading the manuscript and for some interesting discussions.

\section{REFERENCES}

[BB] P. L. Butzer and H. Berens, Semigroups of operators and approximation, Springer-Verlag, New York, 1967.

[C11] Ph. Clément, O. Diekmann, M. Gyllenberg, H. J. A. M. Heijmans, and H. R. Thieme, Perturbation theory for dual semigroups, Part IV, Semigroup Theory and Applications, Lecture Notes in Pure and Appl. Math., vol. 166 Marcel Dekker, New York and Basel, 1989. 
[Cl2] _ A Hille-Yosida type theorem for a class of weakly * continuous semigroups, Semigroup Forum 38 (1989), 157-178.

[DU] J. Diestel and J. J. Uhl, Jr., Vector measures, Math. Surveys, vol. 15, Amer. Math. Soc., Providence, RI, 1977.

[HPh] E. Hille and R. S. Phillips, Functional analysis and semi-groups, rev. ed., Amer. Math. Soc. Colloq. Publ., vol. 31, Amer. Math. Soc., Providence, RI, 1957.

[Ne1] J. M. A. M. van Neerven, Reflexivity, the dual Radon-Nikodym Property, and continuity of adjoint semigroups. II, Indag. Math. 2 (1991), 243-250.

[Ne2] _ On the topology induced by the adjoint of a semigroup of operators, Semigroup Forum 43 (1991), 378-394.

[Pa] B. de Pagter, A characterization of sun-reflexivity, Math. Ann. 283 (1989), 511-518.

[P] A. Pazy, Semigroups of linear operators and applications to partial differential equations, Springer-Verlag, Berlin, Heidelberg, and New York, 1983.

[RSU] L. H. Riddle, E. Saab, and J. J. Uhl, Jr., Sets with the weak Radon-Nikodym property in dual Banach spaces, Indiana J. Math. 32 (1983), 527-541.

[S] H. H. Schaefer, Banach lattices and positive operators, Springer-Verlag, Berlin, Heidelberg, and New York, 1974.

Centre for Mathematics and Computer Science, P.O. Box 4079, 1009 AB Amsterdam, THE NETHERLANDS

E-mail address: neerven@cwl.nl 